\title{
FORMULATION AND EVALUATION OF GRAPHENE GRAFTED CHITOSAN/POLYANILINE NANOCOMPOSITES FOR CONTROLLED RELEASE OF ANTICANCER DRUG DOXORUBICIN
}

\author{
SUCHISMITA MOHANTY ${ }^{1}$, SUBRATA SARANGI' ${ }^{2}$, GOURI SANKAR ROY ${ }^{*}$
}

${ }^{1,2}$ Department of Physics, Centurion University of Technology and Management, Odisha, ${ }^{*}$ PG Department of Physics, College of Engineering and Technology, Bhubaneswar. Odisha

Email: suchismitam547@gmail.com

Received: 16 Dec 2018, Revised and Accepted: 18 Mar 2019

ABSTRACT

Objective: The purpose of the present study was to functionalized graphene (f-GE) grafted chitosan (CS)/Polyaniline (PANI) with Montmorillonite (MMT) was different feed ratio known as f-GE-g-(CS/MMT-PANI).

Methods: The prepared f-GE-g-(CS/MMT-PANI) was formulated using the solvent casting method. The prepared nanocomposites were characterized by X-Ray Diffraction (XRD), Fourier-transform infrared spectroscopy (FTIR), Scanning electron microscope (SEM), Thermogravimetric analysis (TGA), thermogravimetric (DTG) and swelling in stimulated in the different biological fluid. The model drug Doxorubicin (DOX) was used for controlled drug delivery purpose.

Results: From FTIR result was clearly demonstrated that the model drug DOX did not change in any molecular level at f-GE-(CS/MMT-PANI) (i.e. at $<10 \mathrm{~nm}$ scale). Additionally, in DSC result, DOX was interacted with nanocomposites at scale $>100 \mathrm{~nm}$ level. With CS as the carrier, $60 \%$ of the drug was released in SIF for the initial $120 \mathrm{~min}$ and this increased to $80-82 \%$ with f-GE- $g$-CS/MMT/PANI matrix. But in SGF, CS as the carrier, $46 \%$ of the drug was released in $140 \mathrm{~min}$ and this increased to $78 \%$ with f-GE-g-CS/MMT/PANI. In vitro drug release system was carried out by Korsmeyer Peppas's power law. DOX and other drugs like Doxorubicin (DOX) was presented an exceptional higher drug result in different pH medium.

Conclusion: It was observed that CS/MMT was decreasing less drug release rate compared to f-GE-g-(CS/MMT-PANI). So that it can be clearly understood that f-GE-g-(CS/MMT-PANI) grafted nanocomposites have enhanced drug release activity in different $\mathrm{pH}$ medium.

Keywords: Graphene, Chitosan, MMT, Nanocomposites, Doxorubicin, Drug delivery system

(C) 2019 The Authors. Published by Innovare Academic Sciences Pvt Ltd. This is an open access article under the CC BY license (http://creativecommons.org/licenses/by/4.0/] DOI: http://dx.doi.org/10.22159/ijap.2019v11i3.31397

\section{INTRODUCTION}

Chitosan (CS) is a biopolymer which is used for different purpose like biomedical, cosmetics, pharmaceutical products etc. It is very popular in pharmaceutical and medical filed for its unique properties like biodegradable, non-toxic, biocompatible, antimicrobial activities etc. In the present research, it is used for drug delivery applications [1]. Most of the scientific research publications have been proved; CS can be used for oral drug delivery purpose. But its poor physical properties such as brittleness, poor solubility require improvement for more medical application particular in drug delivery field as carrier matrix. Many researchers have been reported that in chemical and biochemical routes will be possible to improve the expected properties. Among grafting procedure, it is easy to prepare and synthesis of modified chitosan polymer composites with biodegrable nature for different purpose [2].

The functionalized graphene acts as efficient nanofiller in polymer composites to improve its engineering properties and a small quantity of polymer functionalized graphene improve the mechanical, electronic, optical, thermal and magnetic properties significantly. The living radical polymerizations on graphene surface can also produce diverse polymeric architecture promoting graphene from a laboratory to important nanotechnological applications.

Chitosan nanocomposites have been well studied for drug delivery and other applications using MMT nanoclay. But limited studies have been made on chitosan grafted onto graphene for drug delivery purpose [2-6]. Generally the hydrophobicity and hydrophilicity nature of polymer is contributing a lot in controlling the drug release characteristics apart from its flexibility [7-10]. However, this experiment is focused on the preparation of chitosan nanocomposites grafted with graphene for controlled drug release of anticancer drug DOX in different $\mathrm{pH}$ medium.

In the present work, GE grafted CS/MMT-PANI to evaluate the feasibility of loading and release of DOX drug with applicable efficiency and to elucidate the effect of biopolymer addition and MMT clay addition on the rate of DOX drug release.

\section{MATERIALS AND METHODS}

\section{Materials}

Chitosan (Mw $=110 \mathrm{kDa}$, deacetylation degree $>90 \%)$ and graphene (GE) were obtained Himedia. Polyaniline, MMT and Doxorubicin (DOX) was purchased from sigma, India. All other chemicals used were of analytical grade which were commercially available.

\section{Preparation of $\mathrm{CH} / \mathrm{MMT}$ nanocomposites}

Chitosan was dissolved in $2 \% \mathrm{v} / \mathrm{v}$ aqueous glacial acetic acid at a concentration of $2 \mathrm{Wt} \%$. The prepared solution samples were subjected to centrifuging at $2000 \mathrm{rpm}$ to remove the insoluble residual material [11]. In addition, MMT was swelled in $50 \mathrm{ml}$ distilled water by magnetic stirring for $60 \mathrm{~min}$ at $30 \pm 1{ }^{\circ} \mathrm{C}$. Then the prepared chitosan solution with chitosan/MMT Wt ratio of $1 / 1,1 / 2$ and $2 / 1$, followed by stirring for $4 \mathrm{~h}$ under different temperature $\left(40^{\circ}, 50^{\circ}, 60^{\circ}\right.$ and $70^{\circ} \mathrm{C}$.) Then, PANI ( $2 \% \mathrm{wt}$, respect to the solid concentration of matrix) was dispersed in an acetic acid solution $(1 \% \mathrm{v} / \mathrm{v})$ for $24 \mathrm{~h}$ and further treated by ultrasonication (Bandelin D-12,207, Germany) and added into polymeric suspension under agitation for $6 \mathrm{~h}$. After that CS/MMTPANI solutions were filtered and dried by vacuum at $70^{\circ} \mathrm{C}$ for $48 \mathrm{~h}$.

\section{Synthesis of GE grafted with f-GE-g-(CS/MMT-PANI)}

The GE-g-CS/MMT was prepared by the following procedure. CS/MMT-PANI was prepared in $1 \%(\mathrm{v} / \mathrm{v})$ acetic acid. The prepared solution of GE $(5.0 \mathrm{~g})$ was added into nanocomposites (CS/MMTPANI). The reaction mixture of solution was refluxed at $100^{\circ} \mathrm{C}$ for 12 $\mathrm{h}$ and dialyzed with distilled water. Then the mixture of solution was lyophilized for preparation of f-GE-g-(CS/MMT-PANI) powder [11].

Preparation of DOX drug-loaded f-GE-g-CS/MMT (f-GE-gCS/MMT-DOX)

Different masses of DOX $(0.05,0.1,0.15$ and $0.2 \mathrm{~g})$ were dissolved with f-GE-g-CS/MMT-DOX sample, which was prepared under the optimum conditions of temperature and concentrations [12]. The 
mixture was subjected to a magnetic stirring at different reaction temperatures $\left(40^{\circ} \mathrm{C}, 50^{\circ} \mathrm{C}, 60^{\circ} \mathrm{C}\right.$ and $\left.70^{\circ} \mathrm{C}\right)$ for different times $(0.5$, 1,2 and $4 \mathrm{~h}$ ) to affect cross-linking.

\section{Characterization}

Fourier transforms infrared (FTIR spectra)

The FTIR spectra were demonstrated on a Nicolet 8700 spectrometer, in the absorbance range in between $400-4,000 \mathrm{~cm}^{-1}$.

\section{$\mathrm{X}$-ray diffraction (XRD)}

XRD instrument Rigaku, D/Max, 2,500 V, Cu- $\alpha$ a radiation: 1.54056A ${ }^{\circ}$ was used for an experiment. The result was recorded at a temperature of $30^{\circ} \mathrm{C}$.

\section{Scanning electron microscopy (SEM)}

SEM of nanocomposites was demonstrated by S 4300 Hitachi, Illinois, USA. The prepared sample was placed into a specimen holder with a silver plate coated by palladium. At analysis time the accelerator potential was $10 \mathrm{kV}$.

\section{Tharmogravity analysis (TGA)}

TGA was performed on a thermal analyser (TA Instruments, SDT Q600) from $40-800{ }^{\circ} \mathrm{C}$ at a heating rate of $10^{\circ} \mathrm{C} / \mathrm{min}$ under nitrogen atmosphere.

\section{Swelling studies}

This study was performed in both simulated gastric Fluid (SGF) and (simulated intestinal fluid (SIF) in phosphate buffer (PBS) at $37^{\circ} \mathrm{C}$ on copolymer pellets $(100 \mathrm{mg}, 2.55 \mathrm{~mm}$ thickness and $13 \mathrm{~mm}$ diameter). The sample was $25 \mathrm{~mm}$ diameter; $50 \mathrm{~mm}$ height which was immersed $20 \mathrm{ml}$ phosphate buffer. The weights of the swelled sample at predetermined time intervals were calculated after wiping the mesh containing the swelled polymer with a tissue paper. Then a graph was drawn between the degree of swelling $\left(=\left(\mathrm{W}_{\mathrm{t}}-\mathrm{W}_{0}\right) / \mathrm{W}_{0}\right)$ and time, where $W_{t}$ and $W_{0}$ are the weights of the sample after and before swelling, respectively.

\section{In vitro drug dissolution study}

In vitro drug dissolution study was made by Veego Model in SGF and SIF at room temperature with 500 RPM. The amount of model drug released was estimated by UV spectrophotometer in the different time interval. The absorbance of pure DOX drug was calculated to be $254 \mathrm{~nm}$.

\section{Statistical analysis}

The experimental data are measured by one-way analysis of variance (ANOVA). All data analysis was executed using the SPSS Statistics 17.0. All experimental data were introduced as a mean value with its standard deviation (mean \pm SD). $P$-values less than 0.05 were recommended to be statistically significant.

\section{RESULT AND DISCUSSION}

As shown in fig. 1(a,b) chitosan was first reacted with MMT and PANI in the presence of acetic acid to functionalize partial amino groups of the CS/MMT-PANI. Then CS/MMT-PANI was reacted with $\mathrm{f}-\mathrm{GE}$ in the presence of acetic acid to functionalize partial amino groups of the chitosan with f-GE and obtained f-GE-g-(CS/MMTPANI) and structure of Doxorubicin.
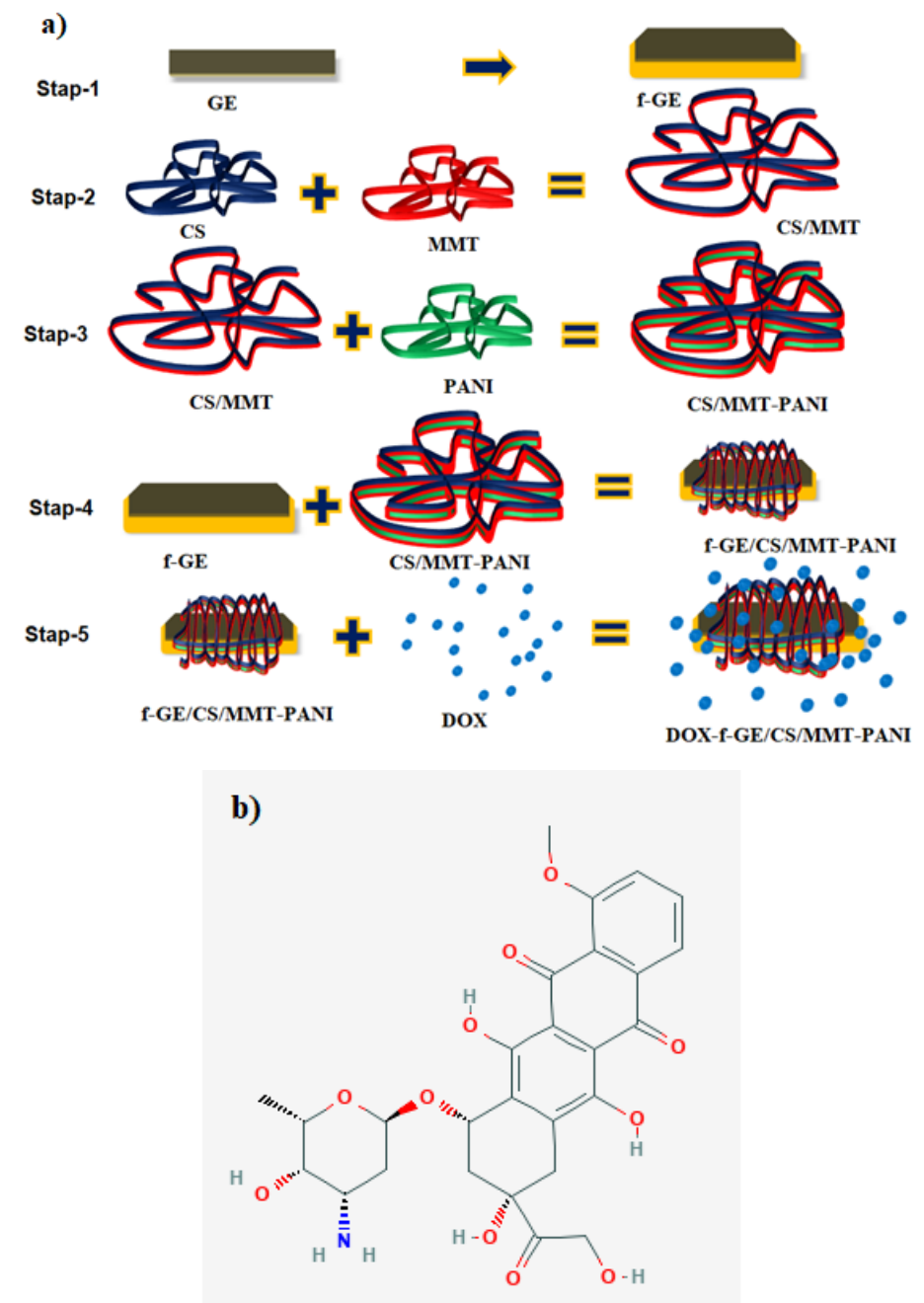

Fig. 1: Schematically representation of f-GE grafted CS/MMT-PANI nanocomposites for controlled release of anticancer drug DOX (a); sturucture of Doxorubicin (b) 


\section{FTIR}

Fig. 2 showed FTIR of pure CS, a broad-OH stretch absorption band between 3500 and $3100 \mathrm{~cm}^{-1}$ and the aliphatic $\mathrm{C}-\mathrm{H}$ stretch between 2345 and $3312 \mathrm{~cm}^{-1}$. The peak at $1505 \mathrm{~cm}^{-1}$ represented the-C-O stretch of the primary alcoholic group $\left(-\mathrm{CH}_{2}-\mathrm{OH}\right)[13]$.

CS/MMT-PANI, the broad peak at $3449 \mathrm{~cm}^{-1}$ (interlayer and interlayer H-bonding upon stretching), $3425 \mathrm{~cm}^{-1}$ (O-H stretching), $2413 \mathrm{~cm}^{-1}(\mathrm{O}$ $\mathrm{H}$ bending), $943-560 \mathrm{~cm}^{-1}$ (Si-O bending vibrations), $1132 \mathrm{~cm}^{-1}$ (Si-O stretching vibrations). At $1534 \mathrm{~cm}^{-1}$ (protonated amine group) of $\mathrm{CH}$ is transformed towards the lower frequency value which indicated an electrostatic interaction between an amino group of CS and the negatively charged sites in the MMT structure [14].

The f-GE-g-CS/MMT/PANI showed both patterns of CS/MMT and GE. The expected peaks of the chitosan backbone was at $3449 \mathrm{~cm}^{-1}$ and $2365 \mathrm{~cm}^{-1}$ (-OH stretching and-C-O stretching), respectively. Further, the absorption peak of the GE vibration of CS at $884.9 \mathrm{~cm}^{-1}$ and the characteristic peak of GE at $967 \mathrm{~cm}^{-1}$ both appeared.

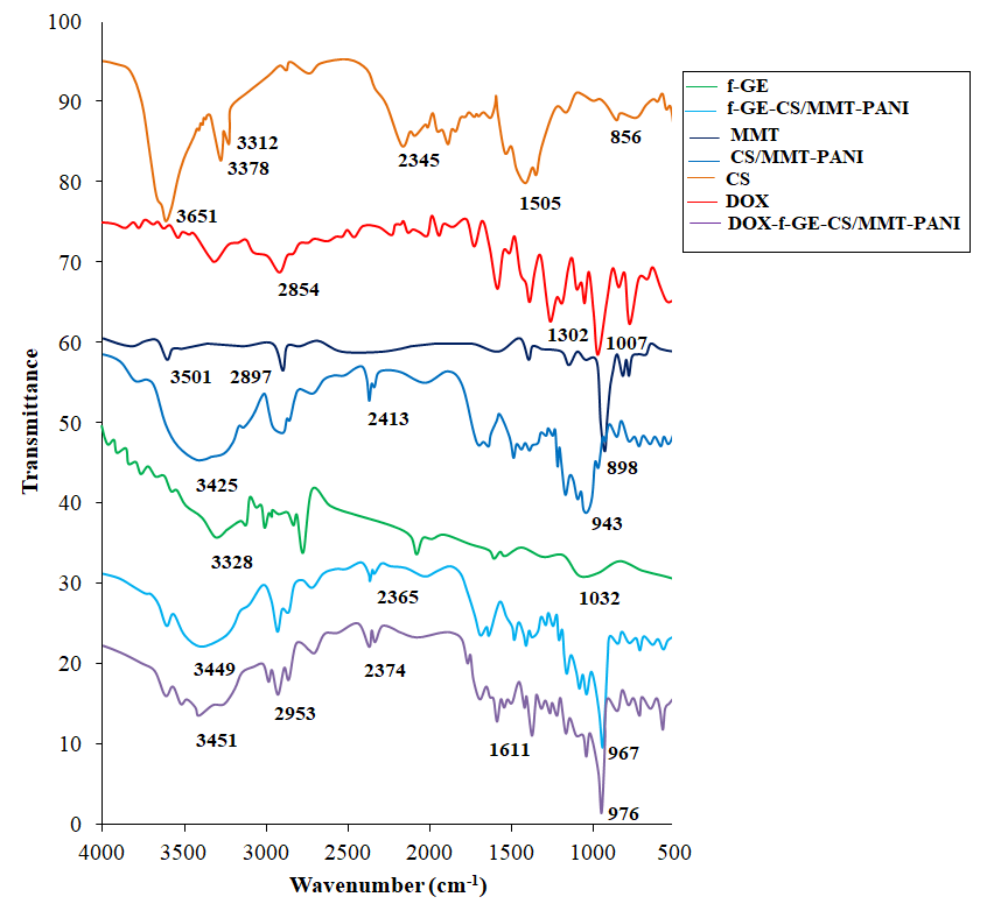

Fig. 2: FTIR of CS, DOX, MMT, CS/MMT-PANI, f-GE- $g$-CS/MMT, DOX-f-GE-g-CS/MMT

XRD

Fig. 3 showed XRD of pure CS, a characteristic peak at $2 \theta=18.95^{\circ}$ and MMT shows a peak at $2 \theta=8.54^{\circ}$ in fig. $2 \mathrm{~b}$ [13]. XRD spectra of $\mathrm{f}$ GE-CS and f-GE-CS/MMT-PANI showed broad single peak pattern at
$2 \theta$ values of $19^{\circ}, 25^{\circ}, 37^{\circ}, 43^{\circ}$ and $45^{\circ}$ respectively, indicating crystallinity and considerably more crystalline than chitosan. It can be hypothesized that the increase crystallinity of f-GE- $g$-CS and f-GE$g$-CS/MMT is due to deformation of the strong bonds in the chitosan backbone as the amino groups are functionalized by f-GE [14].

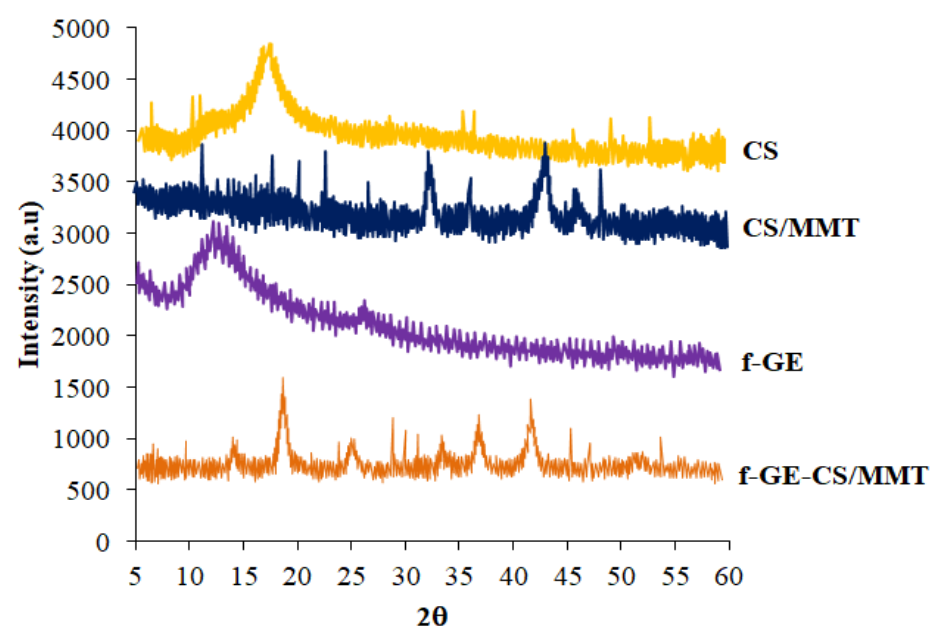

Fig. 3: XRD of CS, DOX, MMT, CS/MMT, $f$-GE- $g$-CS/MMT, DOX-f-GE- $g$-CS/MMT

\section{SEM}

As shown in fig. 4, $\mathrm{f}$-CS/MMT-PANI a nanocomposite was studied by SEM. CS was intercalated structures present due to the interaction of
MMT nanoclay and PANI within the polymer composite. The intercalated structure was found in GE-g-CS/MMT-PANI nanocomposites due to the electrostatic interaction of $\mathrm{f}-\mathrm{GE}$ and CS/MMT-PANI, respectively. 


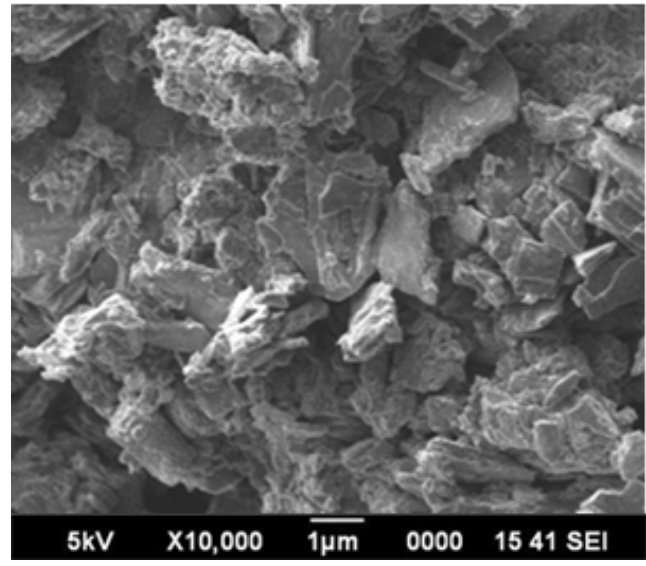

Fig. 4: SEM of f-GE- $g$-CS/MMT-PANI
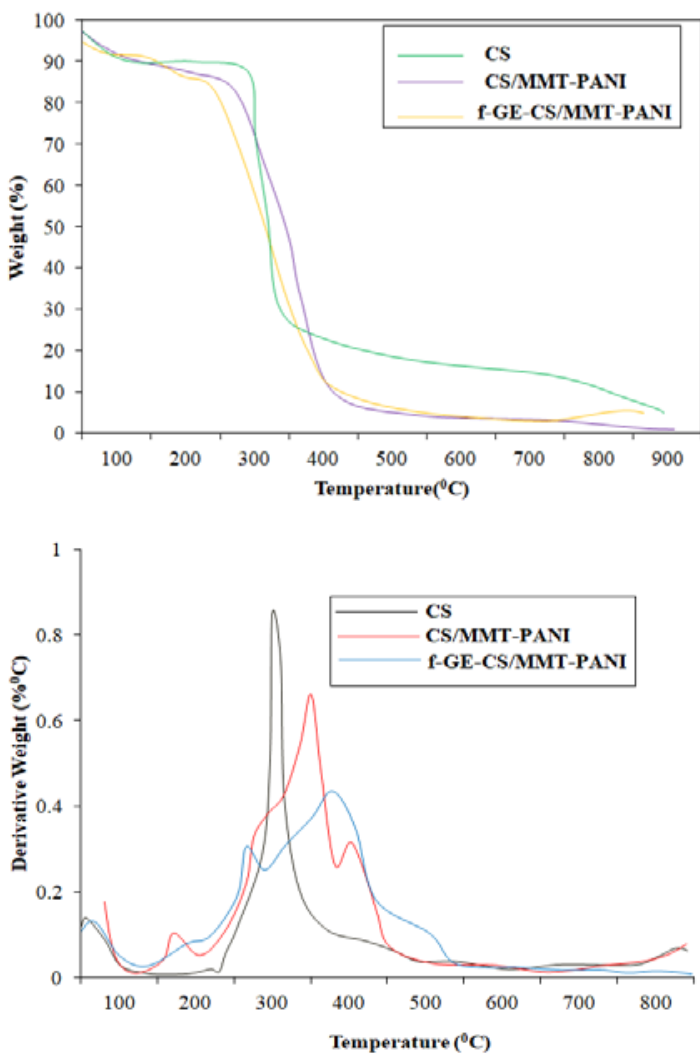

Fig. 5a: TGA of CS, CS/MMT and f-GE- $g$-CS/MMT, b: TG traces of CS, CS/MMT-PANI and GE-g-CS/MMT-PANI

\section{TG and DTG}

TG and DTG thermograms of CS and f-GE-g-CS/MMT-PANI recorded in nitrogen atmosphere are displayed in fig. $5 \mathrm{a}$ and $\mathrm{b}$, respectively. The weight losses around $100^{\circ} \mathrm{C}$ in CS and f-GE- $g$-CS/MMT-PANI are assigned to loss of volatile impurities and moisture. The TG/DTG of graft copolymer displayed three step degradation in the temperature range between $150^{\circ}-900{ }^{\circ} \mathrm{C}$. The weight loss in the temperature range $200^{\circ}-500^{\circ} \mathrm{C}$ with maximum weight loss around $273^{\circ} \mathrm{C}(51.34 \%)$ is attributed to chitosan backbone degradation [14]. The weight loss around $171^{\circ} \mathrm{C}$ is more likely to be attributed to degradation of f-GE fragment on the CS backbone with terminal double bonds. The weight losses observed around $273^{\circ}$ and $381^{\circ} \mathrm{C}$ are due to onset degradations of CS moiety, and poly CS residue with saturated terminal ends and CS moiety, respectively. A degradation step noticed around $387^{\circ} \mathrm{C}$ in CS not seen in the copolymer and the observed residual weights of $7.1 \%$ and $1.3 \%$ at $820^{\circ} \mathrm{C}$ in the $\mathrm{TG}$ traces of CS and CS and f-GE- $g$-CS/MMT, respectively, collectively reveals that the graft copolymer with a decreased thermal stability degrades faster compared to CS. This was also corroborated by the residual mass of $7.1 \%$ at $820^{\circ} \mathrm{C}$ in the TG traces of the polymer blend of CS and f-GE- $g$-CS/MMT (fig. $5 a$ and b). The degradation behavior of the blend, CS and the graft copolymer are quite different indicating that the TG traces of graft copolymer displayed is not a physical mixture of CS and the homopolymers. Different thermal degradation behavior of the graft copolymer compared to that of the blend also revealed that the grafting had occurred $[16,17]$.

\section{Solubility study}

Fig. 7 exhibited the transmittance of f-GE- $g$-CS/MMT-PANI in water with various $\mathrm{pH}$ compared with chitosan and GE- $g$-CS at $5 \mathrm{mg} / \mathrm{ml}$. It was found out that the water solubility of CS, f-GE- $g$-CS and f-GE- $g$ CS/MMT decreased with the increase of pH. CS, f-GE- $g$-CS, f-GE-g$\mathrm{CS} / \mathrm{MMT}$ showed excellent water solubility at low $\mathrm{pH}$ due to protonation of amino groups. At the $\mathrm{pH}$ range of 4.0-9.0, the f-GE- $g$ CS/MMT showed better water solubility than chitosan and f-GE- $g$ $\mathrm{CS}$, which indicated enhances the solubility of chitosan.

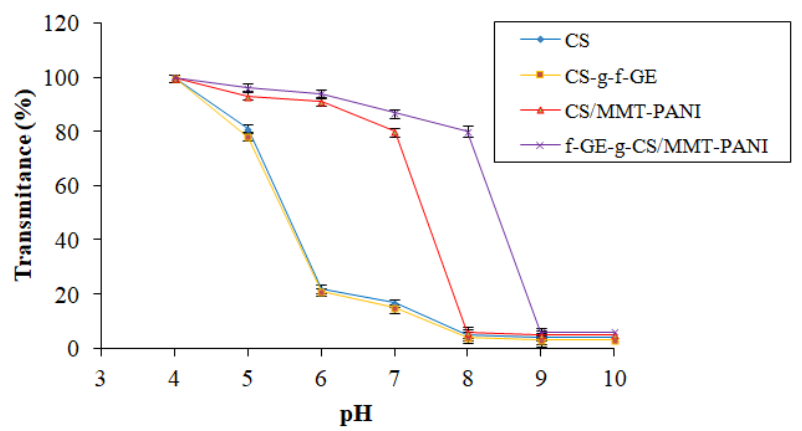

Fig. 7: Solubility study of CS, CS/MMT, CS-g-GE, GE- $g$-CS/MMT; $\mathrm{n}=3$ and data are given in mean $\pm \mathrm{SD}$
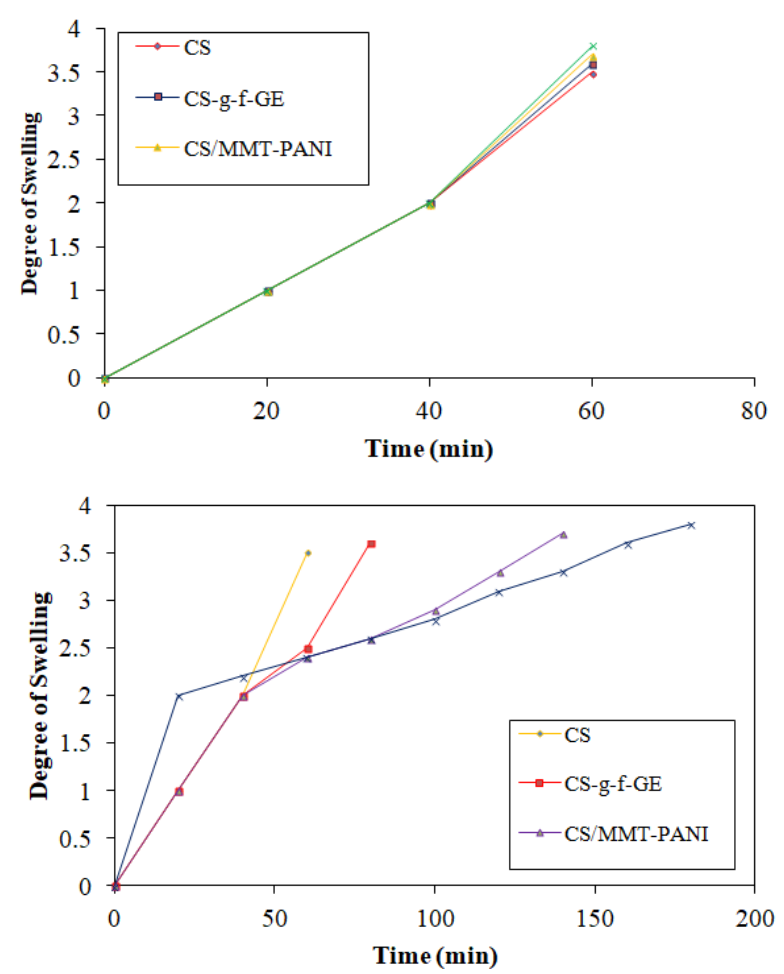

Fig. 8: Degree of swelling of graft copolymer in SGF (a) and SIF (b) at $37^{\circ} \mathrm{C} ; \mathrm{n}=3$ and data are given in mean $\pm \mathrm{SD}$ 


\section{In vitro swelling studies}

To evaluate this effect, swelling studies were carried out with CS/MMT-PANI and f-GE- $g$-CS/MMT-PANI in SGF and SIF at $37{ }^{\circ} \mathrm{C}$ [15]. The degree of swelling in SGF and SIF for f-GE-g-CS/MMT-PANI polymers prepared with increasing concentrations of is given in fig. $8 a$ and $b$. The degree of swelling was significantly different in SIF and SGF for a matrix of the typical composition. In SIF, the rate of swelling is more than that in SGF, and it attains the equilibrium value within $45 \mathrm{~min}$. But in SGF the equilibrium swelling rate was attained only after $160 \mathrm{~min}$. The greater extent of swelling in SIF compared to that in SGF may be more likely due to enhanced hydrogen bonding between GE- $g$-CS/MMT and SIF through the formation of carboxylate anion (-COO).

\section{Dissolution study}

F-GE-g-CS/MMT matrices were synthesized with various concentrations of f-GE in the feed. Both in SIF and SGF the drug release rate was greater for pure CS than that observed for grafted CS/MMT-PANI [15]. This was attributed to the burst release of the drugs due to tablet breaking after immersing in biofluids. For the same drug, the release rate is more in SIF than in SGF (fig. 9a). This is more likely attributed to the greater degree of swelling of the matrix in SIF than in SGF. But grafting decreased the drug release in both the fluids. This is more likely attributed to the enhanced drugpolymer interaction through $\mathrm{H}$-bonding between- $\mathrm{COOH}$ and-OH groups of f-GE- $g$-CS/MMT and-OH group of the drug as supported by DSC (fig. 6). With CS as carrier, $60 \%$ of the drug was released in SIF for the initial 120 min and this increased to $80-82 \%$ with f-GE-gCS/MMT/PANI matrix. But in SGF, CS as the carrier, $46 \%$ of the drug was released in $140 \mathrm{~min}$ and this increased to $78 \%$ with $\mathrm{f}-\mathrm{GE}-g$ CS/MMT/PANI

\section{Drug release mechanism}

To understand and analyze the mechanism of in vitro drug release from nanocomposites by Korsmeyer-Peppas's equation [23, 24],

$$
\frac{\mathrm{M}_{\mathrm{t}}}{\mathrm{M}_{\infty}}=\mathrm{kt}^{\mathrm{n}}
$$

Where $M_{t} / M_{\infty}$ is the fractional release of drug at time' $t$ ' or fractional uptake of fluid in swelling in the absence of drug $\left(\mathrm{M}_{\mathrm{t}} / \mathrm{M}_{\infty}\right.$, amounts of model drug released at the time ' $t$ ' respectively) was used. The release mechanism of the drug was explained by Peppas equation. The slope of the linear plot $\log \mathrm{M}_{\mathrm{t}} / \mathrm{M}_{\infty}$ versus $\log \mathrm{t}$, for drug release $<50 \%$ gives the power law exponent $(\mathrm{n})$ value. The equation $\mathrm{n}=0.5$ (diffusionFickian controlled drug release) and $n=1$ (swelling-controlled drug release or non-Fickian) where is ' $n$ ' anomalous transport kinetics. The observed ' $n$ ' values for typical release profiles of DOX were $<0.5,>0.5$ and $>1.0$. These ranges of ' $n$ ' values indicate a combined mechanism of pure diffusion and non-Fickian for the drug release with the grafted GE-g-CS/MMT/PANI matrix both in SGF and SIF.
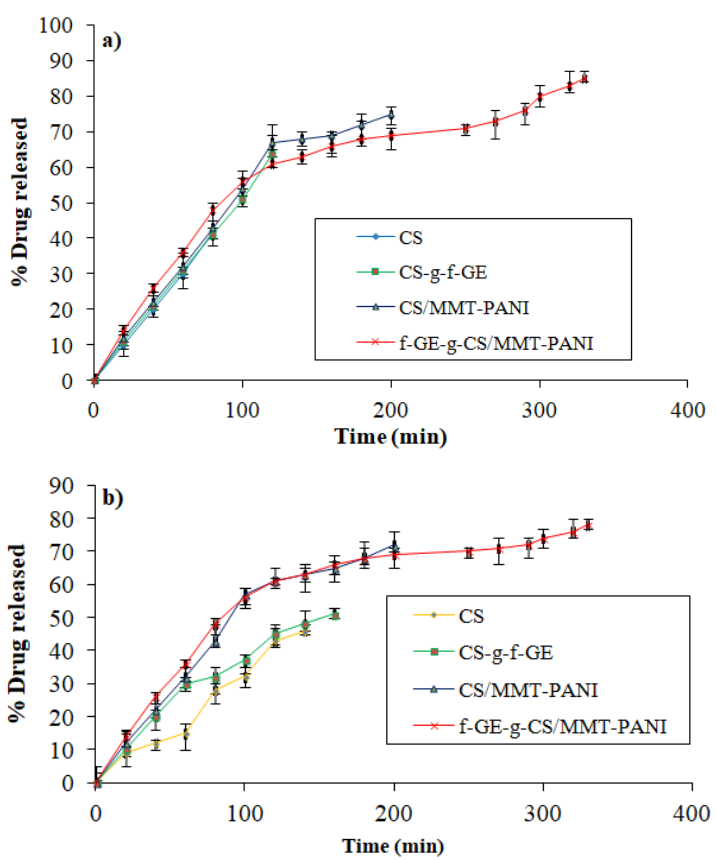

Fig. 9: Release profiles of model drug DOX in SIF (a) and SGF (b) at $37^{\circ} \mathrm{C}$, with graft copolymer carriers $\mathrm{CS}$, CS/MMT, CS-g-GE, GE$g$-CS/MMT/PANI; $\mathrm{n}=3$ and data are given in mean $\pm \mathrm{SD}$

Table 1: Release kinetic parameters of films of DOX $(n=3)$

\begin{tabular}{lll}
\hline Sample & Korsmeyer peppas \\
\cline { 2 - 3 } & $\mathbf{r}^{2}$ & $\mathbf{n}$ \\
\hline CS & 0.9761 & 0.4131 \\
CS/MMT & 0.9831 & 0.4221 \\
CS-g-GE & 0.9758 & 0.5143 \\
GE- $g$-CS/MMT/PANI & 0.9956 & 0.6152 \\
\hline
\end{tabular}

${ }^{*} \mathrm{CS}$ molecular weight in $\mathrm{kDa} . \mathrm{n}=3$ and data are given in mean $\pm \mathrm{SD}$, Where CS: Chitosan, GE: Graphene, PANI: Polyaniline, MMT: Montmorilonite, DOX: Doxorubicin

\section{CONCLUSION}

The nanocomposites f-GE-g-CS/MMT has been synthesized and characterized for controlled released of DOX for oral drug delivery. The drug release rate was greater in SIF than in SGF due to enhanced matrix swelling in SIF and lower with f-GE-g-CS/MMT carrier compared to $\mathrm{CH}$. In SGF the initial drug release rate was sluggish but started increasing rapidly after $120 \mathrm{~min}$ due to increased segmental mobility. Increasing the concentration of f-GE in the feed enhanced the drug release rate with the corresponding grafted CS as a carrier due to enhanced polymer-biofluid interaction.

\section{AUTHORS CONTRIBUTIONS}

All the author have contributed equally

\section{CONFLICTS OF INTERESTS}

All authors have none to declare

\section{REFERENCES}

1. Majeti NV, Kumar RA. Review of chitin and chitosan applications. React Funct Polym 2000;46:1-27.

2. Marguerite R. Chitin and chitosan: properties and applications. Prog Polym Sci 2006;31:603-32.

3. Chen YZ, Huang YK, Chen Y. Novel nanoparticles composed of chitosan and $\beta$-cyclodextrin derivatives as the potential insoluble drug carrier. Chin Chem Lett 2015;26:909-13.

4. Ye YJ, Wang Y, Lou KY. The preparation, characterization, and pharmacokinetic studies of chitosan nanoparticles loaded with paclitaxel/dimethyl- $\beta$-cyclodextrin inclusion complexes. Int J Nanomed 2015;10:4309-19.

5. Yang Y, Gao J, Ma X. Inclusion complex of tamibarotene with hydroxypropyl- $\beta$-cyclodextrin: preparation, characterization, in vitro and in vivo evaluation. Asian J Pharm Sci 2016;12:187-92.

6. Matsubara K, Ando Y, Irie T. Protection afforded by maltosylbeta-cyclodextrin against alpha-chymotrypsin-catalyzed 
hydrolysis of a luteinizing hormone-releasing hormone agonist, buserelin acetate. Pharm Res 1997;14:1401-5.

7. Manakker FVD, Vermonden T, Nostrum CFV. Cyclodextrinbased polymeric materials: synthesis, properties, and pharmaceutical/biomedical applications. Biomacromolecules 2009;10:3157-75

8. Maestrelli F, Gonzalez Rodriguez ML, Rabasco AM. Effect of preparation technique on the properties of liposomes encapsulating ketoprofen-cyclodextrin complexes aimed for transdermal delivery. Int J Pharm 2006;312:53-60.

9. Petter RC, Salek JS, Sikorski CT. Cooperative binding by aggregated mono-6-(alkylamino)-betacyclodextrins. J Am Chem Soc 1989;112:3860-8.

10. Yuan ZT, Ye YJ, Gao F. Chitosan-graft- $\beta$-cyclodextrin nanoparticles as a carrier for controlled drug release. Int J Pharm 2013;446:191-8.

11. Ebru G, Dilay $P$, Cüneyt $H$, Ünlü $O$. Synthesis and characterization of chitosan-MMT biocomposite systems. Carbohydr Polym 2007;67:358-65.

12. Shahrzad F, Ali AM, Azadeh G, Mojtaba K. Electrospinning of PVA/chitosan nanocomposite nanofibers containing gelatin nanoparticles as a dual drug delivery system. J Biomed Materials Res 2015;103:3852-62.

13. Kumari S, Rath PK. Extraction and characterization of chitin and chitosan from (Labeo rohit) fish scales. Procedia Mater Sci 2014;6:482-9.

14. Ebru G, Dilay P, Pestreli C. Synthesis and characterization of chitosan-MMT biocomposite systems. Carbohydr Polym 2007;67:358-65.
15. Thakur G, Singh A, Singh I. Chitosan montmorillonite polymer composites: formulation and evaluation of sustained release tablets of aceclofenac. Sci Pharm 2016;84:603-18.

16. Naguib HF. Chemically induced graft copolymerization of itaconic acid onto sisal fibers. J Polym Res 2002;9:207-11.

17. Isiklan N, Kursun F, Inal M. Graft copolymerization of itaconic acid onto sodium alginate using benzoyl peroxide. Carbohydr Polym 2010;79:665-72.

18. Argin Soysal S, Kofinas P, Lo YM. Effect of complexation conditions on xanthan-chitosan polyelectrolyte complex gels. Food Hydrocolloids 2009;23:202-9.

19. Singh DK, Ray AR. Graft copolymerization of 2-hydroxyethyl methacrylate onto chitosan films and their blood compatibility. J Appl Polym Sci 1994;53:1115-21.

20. Cheung MK, Wang J, Zheng S, Mi Y. Miscibility of poly (epichlorohydrin)/poly (vinyl acetate) blends investigated with high-resolution solid-state 13C NMR. Polymer 2000;41:1469-74.

21. Manley R, St J. Blends of cellulose and synthetic polymers. Cellulose derivatives. ACS Symp Ser 1998;688:253-64.

22. Milosavljevic NB, Milasinovic NZ, Popovic IG, Filipovic JM, Krusic MTK. Preparation and characterization of pHsensitive hydrogels based on chitosan, itaconic acid and methacrylic acid. Polym Int 2011;60:443-52.

23. Korsmeyer RW, Meerwall EV, Peppas NA. Solute and penetrant diffusion in swellable polymers. II. Verification of theoretical models. J Polym Sci Part B: Polym Phys 1986;24:409-34.

24. Ritger PL, Peppas NA. Simple equation for description of solute release II. Fickian and anomalous release from swellable devices. J Controlled Release 1987;5:37-42. 